\author{
Military Technical College \\ Kobry El-Kobbah, \\ Cairo, Egypt
}

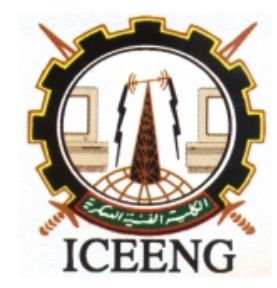

\author{
$7^{\text {th }}$ International Conference \\ on Electrical Engineering \\ ICEENG 2010
}

\title{
Future trend of global navigation satellite systems (GNSS) and innovative applications
}

\author{
By \\ Tarek M.Attia \\ National Telecom Regulatory Authority (NTRA), Egypt
}

\begin{abstract}
:
The purpose of this paper is to provide a general overview of current and future GNSS and various applications. The role played by the current GNSS in our live is set to grow considerably with new demands for more accurate information along with integration into various applications. The real impact of satellite global positioning on society and industrial development became evident and innovative applications will be available when a GALILEO constellation and modernization of both GPS and GLONASS become operational.

There is a need to determine precise ground locations for use in a variety of innovative and emerging applications such as earth observation, rescue applications, and mobilephone technology. Location information is pertinent to a large number of remote sensing applications, some of which support strategic tasks such as disaster management, earth monitoring, protecting the environment, management of natural resources, and food production. With the availability of high-resolution images, some applications will require a location precision down to $1 \mathrm{~m}$. The GNSS provide signals that can serve this purpose.

The traditional fields of application for GPS are shipping, aviation, detection, synchronization of telecommunications networks and security of the objects. However, the market is currently enjoying a growth in demand for electronic car navigation systems which make better use of the road traffic network. Automatic Vehicle Location (AVL) and the fleets' management also appear to be growth rapidly. The advents of new satellite systems as well as the modernization of the current satellite navigation systems and the introduction of new frequencies are expected to contribute to the development of new techniques enabling precise positioning with less complexity and lower cost. The majority of satellite navigation applications are currently based on GPS performances, and great technological developments are spent to integrate satellite-
\end{abstract}


derived information with a number of other techniques in order to reach better positioning precision with improved reliability, accuracy and availability. With all electronics technologies there is an endless challenge aims to improve the performance of GNSS in signal acquisition, signal tracking, multi-path mitigation and interference rejection while at the same time reducing size, power consumption and cost and adding support for more GNSS constellations.

\section{Keywords:}

Global navigation satellite systems, Augmentation systems, Applications

\section{Introduction:}

GNSS are highly effective and a valuable asset to modern society for protecting assets, enhancing safety, managing resources more efficiently, providing entertainment and reducing fear of getting lost. The GNSS, which provide signals that, can be used to accurately locate the position of people and places, and to provide safe navigation information for moving platforms such as ships, aircraft, and automobiles, anywhere on the surface of the Earth and out to near space. GNSS provide information to properly equipped receivers, enabling them to calculate their own position. These receivers, coupled with communication links that transmit the location information, make it possible to know instantly where high value assets and other items of interest are situated.

The world has recognized the potential of having a GNSS and many countries are eagerly establishing systems of their own as a matter of national security and for their economic benefit.

The GNSS of today consists of two satellite constellations, the U.S. GPS and the Russian GLONASS from which military and civilian users can determine their positions free of any charges almost anywhere on Earth. The other systems considered part of GNSS are ground-based and space-based augmentation systems (GBAS and SBAS), like the U.S.A Wide Area Augmentation System (WAAS), and the European Geostationary Navigation Overlay System (EGNOS). Both of them provide enough integrity for GPS signals to allow pilots to use GPS for approaching and landing phases. Civilian and military GPS receivers operate similarly with one major difference. The military has access to higher speed, encrypted signals (10 times more chips per second than the civil signal) that are transmitted by the satellites. This provides two advantages: (1) higher accuracy and interference resistance due to the higher code chipping rate; and

(2) more accuracy because the second frequency enables the user to remove most of the annoying effects on the signal as it passes through the ionosphere. The bending phenomenon occurs when the signal reacts with the ionosphere, but the bending is a well-known function of frequency, so having the range measurements on two frequencies enables the receiver to calculate the bending in the range measurements and 
to correct for most of it. The ability to remove the ionospheric error has been a big factor in commercial GNSS market development and has led to many innovations in civilian receivers, especially for survey applications.

Differential Global Positioning Services (DGPS) were offered in the early 1980s as a means of improving GPS accuracy. Differential correction is required to reduce the error caused by atmospheric interference. This involves placing a GPS receiver on the ground in a known location acting as a static reference point; this is then utilized to identify errors in the satellite data. An error-correction message is transmitted to any other GPS receivers in the local area to correct their position solutions. This real-time differential correction requires radios to transmit the error-correction messages. Alternatively, post processed differential correction can be performed on a computer after the GPS data are collected.

This paper addresses current, future trend and future projects of GNSS. Then focusing the discussion on current and future trend of augmentation systems for achieving the accuracy and integration. The future of user equipment with better accuracy and new signals over the coming years are discussed. Finally the discussion is extended in details on the current and new applications of GNSS.

\section{Current and future trends of GNSS:}

There are currently two global systems in operation: GPS which is owned by the USA and GLONASS owned by the Russian Federation. A third system called GALILEO is under development by the European Community (EC) countries. GPS and GLONASS were designed first to satisfy military requirements. Galileo is the major exception, as it has been funded and designed using civil governmental and private industrial resources. A GPS signal which broadcasts via satellite contains three different bits of information: a pseudorandom code, ephemeris data and almanac data. The pseudorandom code is simply an I.D. code that identifies which satellite is transmitting information. Ephemeris data tells the GPS receiver where each GPS satellite should be at any time throughout the day. Each satellite transmits ephemeris data showing the orbital information for that satellite and for every other satellite in the system. Almanac data, which is constantly transmitted by each satellite, contains important information about the status of the satellite (healthy or unhealthy), current date and time. This part of the signal is essential for determining a position.

There are some factors that can degrade the GPS signal and thus affect accuracy include the following: Ionosphere and troposphere delays, signal multipath, satellite and Receiver clock errors, satellite geometry/shading, orbital errors and Intentional degradation of the satellite signal. The existing systems, GPS and GLONASS, are undergoing modernization and block changes so that they will have operational enhancements by the 2015-2020 time frame [4]. This means that the services provided now will be incrementally improved over time to include better accuracy, stronger 
signals, and higher availability. Other planned systems, primarily Galileo, will add a significant capability to improve reliability and availability for users with receivers which can, for example, make measurements simultaneously from GPS and Galileo.

\section{Current and future trends: GPS System}

The NAVSTAR GPS was developed by the U.S. Department of Defense (DoD). It consists of a constellation of 24 to 27 satellites in operation at any one time (placed in six orbital planes) orbiting the earth at a high altitude (approximately 10,900 miles). Each plane is inclined 55 degrees relative to the equator. The satellites complete an orbit in approximately 12 hours. Up until May 1, 2000, the U.S. government scrambled GPS signals for reasons of national security. This intentional signal degradation was called selective availability (SA) and the positions computed by a single GPS receiver were in error by up to $100 \mathrm{~m}$. But pressure from the civilian GPS user community and other reasons, the government agreed to remove SA. GPS has undergone improvements in all three segments: space, control, and user. In the space segment there have been improvements in signal strength, clock accuracy, and reliability.

In 2000, the USAF began to consider what the future of GPS should be, projecting as far out as 2030. The original plan was to have a competition for the next generation of GPS satellites and control segment, called GPS III. That is still the end goal, but the plan modified several times in order to proceed in stages. This was primarily due to budgetary considerations, Galileo developments, international agreements, and updated potential threat analyses. Since there already were two active contracts for blocks of satellites (GPS Block IIR and GPS Block IIF), the USAF decided first to modify these satellites and the existing control system before starting in the next generation system. This effort is known as GPS Modernization and starting in 2007.

The modified 8GPS IIR (now called GPS IIR-M) spacecraft in orbit have two new signals: L2C for civil users, and a signal for military users known as M-code, or L-M. Interestingly, the L2C signal will be compatible with the Japanese QZSS. The 12 GPS IIF satellites are expected to be deployed during 2010. They have all the same signals as the IIR-M plus a new civil signal called L5 to be used primarily by aviation users and survey receivers. In the late of 2009, L5 (1176.45MHz) carrier signal has been tested in GPS IIF and are used as a civilian signal for aviation to allow for ionospheric correction using only Aeronautical Radio Navigation Service(ARNS) band.

Having civil signals on two frequencies is a major enhancement for civil applications, primarily because a two-frequency receiver can remove many of the errors in the range measurements induced by the ionosphere. The new L2C and L5 of the modernized GPS satellites will enable civil survey receivers to be simplified and more robust [3,4].

The USAF plan calls for a first launch of the next generation spacecraft GPS III in the 2013 time frame. It is believed that benefits from a GPS III satellite will accrue to military users due to considerably increased anti-jam protection and to civil users by the 
addition of another new signal for improvements in accuracy and reliability.

In the control segment there have been improvements in clock, orbit, ionospheric, and tropospheric modeling, enabling better accuracy for all users. The control segment has been expanded to include 11 additional monitor stations deployed throughout the world. The pressure is greatest in the control segment where every new system capability is reflected as a modification to the software that runs the overall GPS. From the military's perspective the most important improvements to be achieved by modernizing GPS are to increase accuracy, improve availability and integrity, and increase resistance to jamming and other electronic warfare measures that could interfere with users. In the later blocks of GPS III, it is expected that there will be a high-power steerable antenna to further concentrate M-code energy in an area of military operations without impacting civilian use outside that area.

\section{Current and future trends: GLONASS System}

The fully deployed GLONASS constellation is composed of 24 satellites in three orbital planes. Each satellite operates in circular 19,100km orbits at an inclination angle of 64.8 degrees, and each satellite completes an orbit in approximately 11 hours and 15 minutes. The spacing of satellites in orbits is arranged so that a minimum of five satellites is in view to users worldwide. The GLONASS constellation provides continuous and global navigation coverage. Each GLONASS satellite transmits a radio-frequency navigation signal containing a navigation message for users. The deployment of the full constellation of satellites was completed in 1996, although GLONASS was officially declared operational on September 24, 1993.

GLONASS has been undergoing a renaissance this decade as the Russians have started new life into the constellation and announced plans to keep the system operational for both military and civilian users. The Russians will improve GLONASS coverage by renewal the constellation and by updating its timing system. With respect to the spacecraft, there has been a second civil signal at the GLONASS L2 frequency since 2003 on what is known as the GLONASS-M satellites. A third civil signal at the GLONASS L3 frequency will be on the newer GLONASS-K satellites. The GLONASS constellation is expected to grow to 24 satellites by 2010[2, 7]. The Russians have stated their intention to have future compatibility between GLONASS, GPS, and Galileo. Similarly, they also intend to have their industry develop receivers that can listen to all three systems. As the end of the decade approaches, the state of the art in digital software receiver design should allow for a competitive product.

\section{Future Trends: GALILEO System}

GALILEO is the global navigation satellite system being developed by an initiative launched by the European Union and the European Space Agency (ESA). GALILEO will be fully operable by 2013. This worldwide system will be interoperable with GPS and GLONASS and will deliver real-time positioning accuracy down to the meter range, which is unprecedented for a publicly available system. Figure (1) below shows 
continuous improvement in accuracy achieved by GPS.

Figure (1): continuous improvement in accuracy achieved by GPS [5].

The fully deployed GALILEO system consists of 30 satellites (27 operational plus three active spares) positioned in three circular medium-earth-orbit (MEO) planes at an altitude of 23,616 km above the Earth, and with an inclination of the orbital planes of 56 degrees in reference to the equatorial plane.

GALILEO will provide a global search and rescue (SAR) function similar to the existing operational Cospas-Sarsat system. To do so, each satellite will be equipped with a transponder that is able to transfer the distress signals from the user transmitters to the rescue coordination center, which will then initiate the rescue operation. The system will also provide a signal to the user, informing the user that the situation has been detected and that help is under way. This feature is new and is considered a major upgrade to the current two systems.

Negotiations with U.S. administration are currently focusing on the shared use of certain frequency bands, which will allow a combined GPS and GALILEO receiver that will be capable of computing signals from both constellations. This will provide for the best possible performance, accuracy, and reliability. The critical issue with the current implementation of GNSS for nonmilitary purposes is that some applications require the system to have special features. These features include service guarantee, liability of the service operator, traceability of past performance, operation transparency, certification, and competitive service performance in terms of accuracy and availability. These features do not currently exist in the current systems. New applications are appearing everyday in this huge market, which is projected to reach at least 3,600 million in 2020. Galileo is configured with several different frequencies that emit signals for different services. There is a free open service along the lines of the GPS Standard Positioning Service and four other services that require a fee. The fee-based services include: a commercial service with an assured level of performance and value-added data accessible via decryption; a Safety-of-Life service with high integrity that meets ICAO and IMO standards; an encrypted Public Regulated Service(PRS) with high interference 
resistance for government customers; and a SAR which guarantees locating a distress signal quickly and accurately $[1,9]$.

\section{Future projects for GNSS:}

China has indicated they intend to expand their regional navigation system, called Beidou into a global navigation system; a program that has been called Compass. This system is proposed to utilize $30 \mathrm{MEO}$ satellites and five geostationary satellites. Having announced they are willing to cooperate with other countries in Compass's creation, it is unclear how this proposed program impacts China's commitment to the international Galileo position system. In late 2006, the Chinese announced that Compass would indeed go forward as a global system for their military and include a civil signal as well. The Indian Regional Navigational Satellite System (IRNSS) is an autonomous regional satellite navigation system being developed by Indian Space Research Organization which would be under the total control of Indian government. The government approved the project in May 2006, with the intention of the system to be completed and implemented by 2012. It will consist of a constellation of 7 navigational satellites by 2012. All the 7 satellites will be placed in the Geostationary orbit (GEO) to have a larger signal footprint and lower number of satellites to map the region. It is intended to provide an absolute position accuracy of better than 20 meters throughout India and within a region extending 2,000 km around it. A goal of complete Indian control has been stated, with the space and ground segment and receivers all being built in India.

The Japanese have moved out smartly in adopting satellite navigation and manages to deploy a QZSS; they would consider deploying a system called the Japanese Regional Navigation Satellite System (JRANS). This would increase the constellation of QZSS to seven satellites, one of which would be geostationary. The satellites were to be in different inclined elliptic orbits so as to always pass over the same ground track, and they were to be phased such that one satellite is always at zenith over Japan. This constellation would provide increased coverage in East Asia and also compatibility with other future GNSS signals [2].

\section{Augmentation systems (SBAS and GBAS) and future trend}

In addition to the global component of GNSS, in many areas of the world there are augmentation components like SBAS as well as GBAS regional components.

There are plans in several countries to build SBAS and then to expand them into either full GNSS or regional navigation systems, thus giving each respective country control over their own navigation system while contributing to improved seamless global navigation for aviation users and others.

WAAS has been operational since 2003 and has had improvements such as additional geosynchronous satellites and reference stations deployed. The FAA had been certified WAAS for Category I Instrument Landing System approaches to airports [1]. WAAS consists of a series of reference/monitor stations that in effect, keep an eye on GPS. 
Pseudo range (PR) measurements taken at these monitor stations are tagged and sent continuously to a master control station. There, all the PR measurements are analyzed to detect any anomalies in the operation of any of the GPS satellites. Such anomalies must be communicated within a very short time to all aircraft using GPS as a navigation aid, whether in flight, on approach, or landing. The required integrity of navigation performance dictates a warning time of just a few seconds as the plane begins its approach to an airfield. Solving that communications problem without impacting the cost of avionics has been achieved by WAAS through the use of transponders aboard synchronous communications satellites.

Instead of requiring a new radio or modem aboard the aircraft to receive the WAAS broadcast, the signal communicated over the synchronous WAAS satellite is in the form of a GPS satellite signal using codes that are not assigned to the 30 satellite GPS constellation. The WAAS data message contains not only warning information on any of the GPS satellites that are out of specification or unusable, it also contains differential corrections for the GPS satellites that are in view of the user. Most modern GPS receivers have the ability to receive WAAS broadcasts. They treat the WAAS signal as just another satellite pseudo range and use the differential corrections to improve overall accuracy. The integrity warning message is primarily for the aviation sector.

EGONS was accepted by the ESA in June 2005 and officially entered into operational phase with the provision of open service by the end of 2009[1, 2]. EGONS has demonstrated excellent performance with augmentation of GPS accuracy reached to one to two meter at an availability level above $99 \%$ and will start providing safety of live for aviation around mid 2010[1]. EGNOS operates similarly to WAAS. There are 34 reference/integrity monitoring stations, six uplink stations to transmit to three geosynchronous satellites, and four mission control centers to provide redundancy. In addition to GPS integrity, EGNOS is planning to include integrity message information on the reliability of GLONASS and eventually on Galileo.

Many other countries are actively considering how to participate in the worldwide effort to ensure integrity in navigation, particularly for air services.

The SBAS of Japan is part of the Multi-Functional Transport Satellite (MTSAT) Program. MTSATs are synchronous communications, weather, and aircraft surveillance satellites. They will also be used to transpond integrity and other information to users as part of the Japanese SBAS.

India has a significant aerospace industry and capability. To foster its growth and to join in the world's tendency for safer air travel, they have begun the GPS and Geo Augmented Navigation (GAGAN) Program. This is a pure SBAS like WAAS and EGNOS. In fact, the coverage of GAGAN is such that it will bridge the gap between EGNOS to the west of India and MSAS to the east. The first phase of the program was a technology demonstration that includes eight reference receivers. The Master Control Station is in Bangalore, where one of the receivers is collocated. The transponder 
package was aboard the GSAT-4 geostationary satellite after launch and testing, the system will become operational [2].

Canada's SBAS is based on an extension of the U.S. WAAS coverage by adding reference stations throughout Canada and linking them to U.S. WAAS control stations. GBAS describe a system that supports augmentation through the use of terrestrial radio messages. As with the satellite based augmentation systems detailed above, ground based augmentation systems are commonly composed of one or more accurately surveyed ground stations, which take measurements concerning the GNSS, and one or more radio transmitters, which transmit the information directly to the end user. Generally, GBAS networks are considered localized and transmitting in the MF, HF, VHF or UHF bands. There are many government-sponsored commercial and private GBAS. Several of the first DGPS were for the military applications. The major difference between GBAS and SBAS is its way of communicating differential corrections and integrity warnings between reference/monitor stations and users.

\section{Future User Equipment:}

With the advent of all the new signals over the coming years and the new satellite navigation systems and augmentations that will be deployed, there will also be an ample of new user equipment that can take advantage of these signals.

The generic GNSS user equipment has an antenna, receiver, processor, oscillator, frequency synthesizer, display, and batteries. Over time, these components will experience an upgrade in technology, as has been the case throughout the development of GPS user equipment. What is different now is that improvements made to many types of user equipment will have to include the ability to listen to two or more satellite systems and to harmonize differences in their data messages relating to system time and geographic references. An obvious design issue will be the antenna. For civilians this has largely been a case of designing an antenna that is small for handhelds, flush mounted for vehicles and aircraft, and low multipath for reference stations and other applications. But these civilian antennas were for the most part L1 only except for realtime kinematic (RTK) applications. Now they will have to be broader in bandwidth to make use of the new L2 C and L5 signals and the common (with GPS) signals from the other GNSS components.

Receivers developed these days are tending toward becoming true software-based receivers with an analog/digital converter after the RF/IF front-end section followed by a powerful processor that can perform amazing low signal-to-noise ratio detection, correlation, tracking, and demodulation. Having a software-based receiver makes it easier to design a multisystem receiver as long as the RF signals can be received at a level suitable for digital processing [10]. The users of future GNSS can look forward to increased accuracy, faster fixes, and more integration of functions in their equipment. By the time all the planned satellite navigation systems and augmentations are 
operational, user equipment will have evolved into unimaginable complexity of function, simplicity of use, and increased cost effectiveness for the many applications. Manufacturers in many countries are developing the receivers to enable use of satellite navigation systems and augmentations systems. Modern technology rapidly evolves, GPS receivers will continue to shrink in size and they are being used in every conceivable type of electronic device. GPS receiver price will continue to decline as manufacturing costs decrease and production quantities increase. Rechargeable GPS receivers have been realized, miniature fuel cell technology will keep GPS units going and going without frequent battery changes.

Today's GPS receivers are extremely accurate; thanks to their parallel multi-channel design which quick to lock onto satellites when first turned on and they maintain strong locks, even in dense foliage or urban settings with tall buildings. Newer GPS receivers with WAAS and EGONS capability can improve accuracy to less than three meters on average. No additional equipment or fees are required to take advantage of WAAS. Users can also get better accuracy with DGPS.

\section{GNSS applications:}

The main segments of the GNSS market are military and commercial, where the commercial market is further divided into the various application fields. A possible classification for these segments is: commercial aircraft and aviation; marine and recreation; automobile navigation; vehicle tracking; cell phones; survey and other highaccuracy applications such as agriculture, mapping and GIS; timing and reference stations.

\section{Maritime Applications:}

There are many more ships and boats than there are aircraft on this planet, and many of them transit oceans where navaids are sparse at best. This market segment has whole heartedly adopted satellite navigation and has been using it for more than 40years.

Ships engaged in international trade must be equipped with a certain minimum set of equipment for communications and navigations. The standards that the equipment must meet are codified by agreement of the member states of the Commercial coastal and inland vessels.

There are many applications for extremely accurate positioning for seismic survey and oil and gas exploration activities as well as in dredging, buoy laying, and maintenance. The rise of worldwide terrorism stimulates the development of means for tracking large container ships as they sail on the sea. GPS plays an important role in these kinds of systems, which also rely on satellite communications and electronic tagging.

With the growth in world trade and the need for security, vessel tracking, especially of cargo carriers, has become an industry. Standard DGPS and FM radio communications were used to locate ships and transmit their locations to a display at the reference station. The ships paths were also displayed onboard. 
The International Maritime Organization's (IMO) in May 2006 adopted new regulations for Long Range Identification and Tracking (LRIT) system, together with associated performance standards and functional requirements. Figure (2) shows the LRIT system architecture.

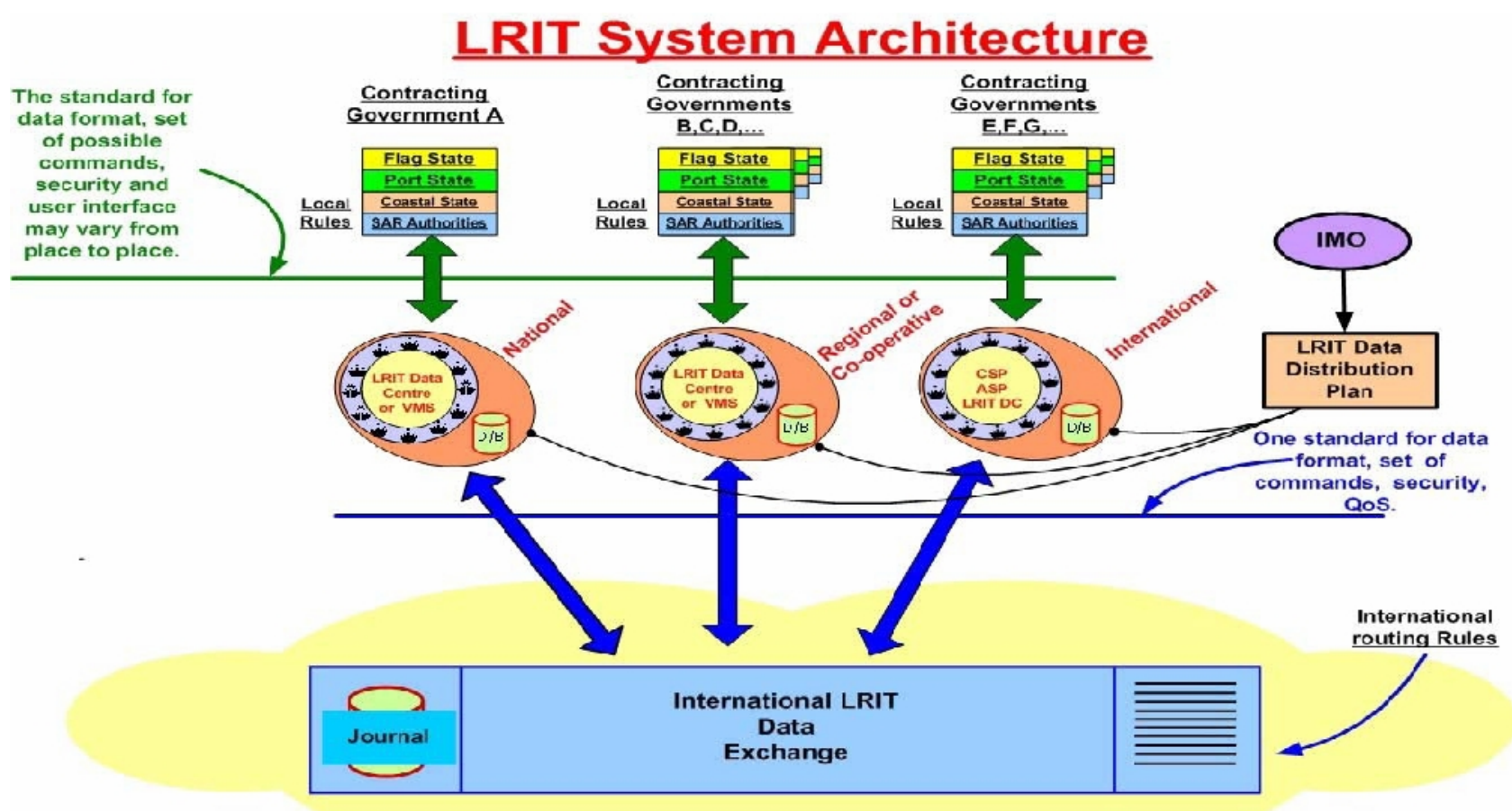

Figure( 2): LRIT system architecture

LRIT introduces as a mandatory requirement for the ships on international voyages and Mobile offshore drilling units. From 2009, LRIT enters into operation as one application of GNSS for protecting ships from piracy and environmental protection and for sharing LRIT information for security and search and rescue (SAR) purposes amongst SOLAS Contracting Governments. The LRIT information to be transmitted periodically or on demand by ships includes Ship's identity, Location, Date and time of the position [7].

Automatic Identification System (AIS) is another system used by ships and VTS principally for identification and locating vessels to avoid collision. AIS helps to resolve the difficulty of identifying ships when not in sight (e.g. in fog) by providing a means for ships to exchange identification, position, course, speed, and other ship data with all other nearby ships and VTS stations. It works by integrating a standardized VHF transceiver system with GPS receiver, and other navigational sensors on board ship [7].

\section{Air and Space Applications:}

Aviation users have much to benefit from the use of GNSS and its augmentations. This market has been limited by two factors: government regulation and economic conditions in the airline industry, such as the problem of rising fuel costs. Regardless, there is a worldwide push by aviation authorities, especially in the USA and in Europe, to 
increase traffic capacity in the airspace and to improve efficiency at airports.

Use of GPS for approaching and landing requires a very high level of integrity. In order to reach that level, a continuous check on the performance and the quality of the information being derived from GPS measurements would have to be accomplished by independent systems such as WAAS and EGNOS [2].

Modernized GPS also is accommodating greater civil aviation use with its forthcoming L2C, L5 signal on GPS IIF and GPS III. It is hoped that in the 2010-2015 time frame there will be a seamless system as far as air traffic management is concerned so that aircraft can use their standardized equipment to fly safely in anyone's civil airspace with the same level of confidence in their navigation and positioning [4].

Commercial airline pilots view satellite navigation as a tool to increase safety of operations through better knowledge of where an aircraft is located at all times. While safety is the major concern of the airlines, fuel cost savings through more efficient routing and direct aircraft flights through better air-traffic management, more efficient ground control, fewer flight delays and better airport operations is also very important to them. GPS receivers are common on today's larger airliners. The receivers make use of WAAS broadcasts and also have algorithms buried within their signal processing functions which perform self-monitoring checks on the adequacy of received satellite signals. Somewhat unique to aircraft receivers, this Receiver Autonomous Integrity Monitoring (RAIM) processing looks at all the satellites in view and makes fixes using every possible combination of four satellites. With such redundancy, RAIM can detect a "bad" measurement with only five GPS satellites in view. When there are six satellites in view, RAIM can not only detect the out-of-bounds measurement, it can determine which satellite is causing the poor fix and eliminate it from any further calculations. Coupling RAIM with WAAS information makes for an extremely robust receiver that meets very stringent FAA and ICAO requirements for navigation performance, integrity, availability, and continuity of service. With only GPS satellites to do RAIM, there are many areas of the world during the day are not always visible by six satellites and these are known as RAIM holes. However, when the additional coverage provided by Galileo satellites is added, the RAIM holes will disappear [4].

\section{Land Applications:}

There is no segment with greater potential for growth than the land segment. This field has many different applications and we will demonstrate it in the following sections.

\section{Navigation and Automatic Vehicle Location services}

The market for Fleet Management System (FMS) is expected to grow to over 5.2 million units with yearly service sales crossing the $\$ 2.1$ billion mark through 2009[5].

At present, road accidents generate social and economic costs corresponding to 1.5 to $2.5 \%$ of the gross national product (GNP) of the European Union. Road congestion entails additional estimated costs of around 2\% of the European GNP. Americans drive 
or ride a total of 11 billion miles per day. It was forecast that safety improvements made via the application of GNSS technologies will reduce the annual traffic death rate by about 30\% from current levels and have considerable socioeconomic benefits [5].

Vehicle manufactures now provide navigation units that combine satellite location and road data to avoid traffic jams and reduce travel time, fuel consumption, and therefore pollution. Road and rail transport operators will be able to monitor the goods' movements more efficiently. Taxi companies now use these systems to offer a faster and more reliable service to customers. In addition, police and other government agencies are using GPS tracking systems to monitor the locations of their own vehicles. As in all real-time tracking systems, cell phone or other wireless links are being used in order to transmit location information from the tracked units to the controllers. Urban transit buses are finding application of GPS for schedule maintenance and safety enhancement. The drive toward increasing the capacity of the existing transportation infrastructure has spawned the emerging concept of Intelligent Transportation Systems (ITS) which are meant to modify traffic flow according to demand and other factors.

Another aspect of the ITS involves the automatic collection of highway and other tolls and tariffs. This eliminates the need for vehicles to stop at state lines or at toll booths on toll roads and bridges if their position is being reported and appropriate accounting arrangements are made between the tariff-collecting authority and the vehicle's operator. Another application of vehicle tracking is called geofencing, where a vehicle's GPS is programmed with a fixed geographical area and alerts the fleet operator whenever the vehicle violates the prescribed fence. The GPS monitor/recorder installed in the car keeps a record of the vehicle's speed and places it traveled in case there is a dispute. Automobiles and trucks are being equipped with black box recorders such as those required on commercial aircraft in order to have information about all vehicle systems as well as its speed and location at the time of an accident.

GNSS receivers can be used as a vital tool for law enforcement for keeping track of criminals. Numerous law enforcement agencies rely on GPS for tracking applications, especially for surveilling suspected criminals or recovering GPS-equipped stolen vehicles.

\section{Location-Based Services and Cell phone applications}

One Example of location based services (LBS) is E911 calls in the USA and Canada (E112 in Europe and E110 in Japan). E911 is automatically associates a physical address with the calling party's telephone number, and routes the call to the most appropriate Public Safety Answering Point (PSAP) for that address. The caller's address and information is displayed to the call taker immediately upon call arrival. This provides emergency responders with the location of the emergency.

Wherify Wireless Company in USA offers a cell phone solution called Whereifone, which, for example, allows a parent to monitor a pre-teen's whereabouts and also gives youngsters the ability to hit a panic button whenever they need help. The parent can 
access the child's location by calling the phone or through the Internet. There is also potential for the signal to be used to guide the blind; monitor Alzheimer's sufferers with memory loss; and guide explorers and hikers.

With new services from Verizon Wireless and other carriers, people who are simply walking the streets can have the same kind of voice guidance they have in their cars for a much lower cost. The user would type a destination address into their cell phone. Verizon would calculate their position and an optimum route to their destination and then send a voice message to the user which describes the path they should follow [5].

Cell phone providers inherently have a means of providing value-added services to their customers. From simply transmitting maps and directions to a cell phone user to sending more complex information relating to, for example, traffic, travel, entertainment, and shopping. Cell phone service providers are offering new LBS. In urban canyons and inside buildings it is usually much easier and reliable to receive cell phone signals than it is to receive GNSS signals, so in addition to providing A-GPS signals for improved GPS performance, cell phones can also be a basic element of a device that can be used to locate people such as firefighters in these environments.

\section{Precision and High Accuracy Applications of GNSS}

In many instances ordinary stand-alone GPS accuracy is not sufficient. In these applications the use of DGPS and sometimes even RTK DGPS is needed. For modern GNSS-based navigation tools, the maps used are in a digital format. A digital map has had the symbols geocoded and stored as a data structure within the map database. This data have become available in compact form by purchasing a new DVD or downloads from the Internet or via cell phone.

Surveying systems incorporating GNSS signals will be used as tools for urban development. They can be incorporated into geographical information systems for the efficient management of agricultural land and for aiding environmental protection; this is a critical role of paramount importance to assist developing nations in preserving natural resources and expanding their international trade.

The same type of receivers used for surveying can also be used for other high accuracy applications, such as in the utilities industry to maintain accurate records and maps of the location of their assets [5]. Similarly, the forestry and natural resources industry also makes use of GNSS for fire prevention and control, harvesting, boundary determination, and insect control.

The agriculture and farming industry makes heavy use of GNSS as part of a modern precision farming system includes spraying fertilizer, seed, or insecticide, or directing combine machines exactly where to go to harvest a crop, the application of these materials is becoming an increasingly exacting science.

Others industries that use high accuracy GNSS equipment include the construction industry for machine control and for directing Earth movers and aiding in road building, and the mining industry for assisting in drilling and earth monitoring. 
Some of the most common uses of GPS time are as follows: Internet Protocol and communications network synchronization; frequency reference control; calibration of test instruments; clock synchronization; and time and frequency distribution [5, 8].

\section{Conclusions:}

The role played by the current global navigation satellite systems (GPS and GLONASS) in everyday live is set to grow considerably with new demands for more accurate information along with integration into more applications. The real impact of satellite global positioning on society and industrial development will become evident when GALILEO becomes operational and modernization of GPS and GLONASS.

The vast majority of satellite navigation applications are currently based on GPS performances, and great technological effort is spent to integrate satellite-derived information with a number of other techniques in order to reach better positioning precision with improved reliability.

The availability of two or more constellations will double the total number of available satellites in the sky, therefore enhancing the quality of service and increasing the number of potential users and applications.

The market for high precision GNSS technology is growing and it is expected to continue for a while. There are many exciting applications that remain unexplored. As the performance of GNSS receivers increases, as adding more GNSS constellations and as the cost of GNSS receivers comes down, it is expected that the high precision market will grow dramatically.

\section{References:}

[1] http://www.insidegnss.com, Jan/Feb.2010.

[2] http://www.insidegnss.com, Nov/Dec.2009.

[3] http://www.insidegnss.com, May/June2009.

[4] http://www.insidegnss.com, Jan/Feb.2007.

[5] Len Jacobson, GNSS Markets and Applications, Norwood, MA: Artech House, 2007.

[6] Kaplan, E., and C. Hegarty, (eds.), Understanding GPS: Principles and Applications, 2nd ed., Artech House, 2006.

[7] IMO Web site, http://www.imo.org/home.asp.

[8] http://www.cellularnews.com, September 28, 2006.

[9] Galileo Web site, http://europa.eu.int/comm/dgs/energy_transport/.

[10] James Bao-Yen Tsui, "Fundamentals of Global Positioning System Receivers A Software Approach", John Wiley \& Sons, Inc. 2000. 